\title{
Metodología numérica para resolver ecuaciones del modelo elíptico de un superconductortipo II anisótropo
}

\author{
Omar A. Hernández-Flores y Carolina Romero-Salazar \\ Escuela de Ciencias, Universidad Autónoma "Benito Juárez" de Oaxaca \\ Apdo. Post. núm. 76, Oaxaca de Juárez, Oaxaca, C.P. 68120, México. \\ omar22121972@gmail.com, carolainflais@gmail.com
}

\begin{abstract}
Resumen
Con base en una descripción macroscópica de un material superconductor tipo II anisótropo, se plantea la metodología numérica para modelar la inducción magnética de estos materiales en estado crítico. En este caso, se emplea el modelo elíptico de estado crítico para describir un superconductor tipo II anisótropo en la geometría paralela. En este trabajo describimos cómo se resuelve el problema matemático para hallar la distribución de la inducción magnética en el material y que consiste en resolver un sistema de ecuaciones diferenciales ordinarias; para ello se emplean bibliotecas estándar del software llamado MATLAB. Particularmente se utilizaron dos rutinas, una de ellas se emplea para dar una solución inicial gruesa que después se refina con la segunda rutina. Los autores cuentan con amplia experiencia en el estudio macroscópico de las propiedades magnéticas de superconductores tipo II así como publicaciones internacionales en esta área de estudio.
\end{abstract}

Palabras clave: anisotropía, corriente crítica, estado crítico, método de líneas, superconductor tipo II.

\section{Summary}

Based on the macroscopic description of a type II anisotropic superconducting material, this paper presents numerical methods to model the magnetic induction of these materials in a critical state. In this case, an elliptical model of the critical state is used to describe a type II anisotropic superconductor in terms of parallel geometry. It describes how the mathematical problem is sol- ved in order to find the distribution of magnetic induction in the material. This consists of solving a system of ordinary differential equations. In order to do this, the standard libraries of MATLAB software were used. Two routines were especially used, the first of which gives an initial rough result which is then refined with the second routine. The authors have ample experience in the macroscopic study of the magnetic properties of type II superconductors as well as international publications in this specialized study area.

Key terms: anisotropy, critical current, critical state, method of lines, type II superconductor.

\section{Introducción}

En la actualidad existe tecnología que emplea superconductores tipo II tales como los dispositivos electrónicos llamados Superconducting Quantum Interference Devices y circuitos integrados; en transporte como los trenes de levitación magnética; en medicina para la obtención de imágenes a través de resonancia magnética; en procesos industriales; en ciencia básica a través de la construcción de imanes y en producción de energía a través de cables o transformadores, por citar algunos ejemplos. En toda aplicación tecnológica, los superconductores están sometidos a campos magnéticos en configuraciones no triviales, por lo que los modelos fenomenológicos son útiles para predecir o explicar su comportamiento magnético.

El modelo elíptico de estado crítico -un modelo fenomenológico- describe el comportamiento de los campos electromagnéticos en una placa infinita superconductora en la geometría paralela (Romero-Salazar, 2003), sometida a cam- 
pos magnéticos variables en magnitud y dirección. El modelo ha sido exitoso al cotejar sus resultados teóricos con mediciones experimentales, tanto de materiales granulares y materiales con anisotropía estructural, así como para describir el corte de líneas de flujo.

Sin embargo, la descripción y un análisis a fondo de la metodología numérica empleada para hallar los campos electromagnéticos, ha estado ausente en trabajos previos de los autores. En este trabajo nos centramos en mostrar cómo se resuelve numéricamente el sistema de ecuaciones diferenciales parciales con dos puntos fijos a la frontera y se dan detalles adicionales de la metodología numérica.

\section{Teoría}

El sistema de estudio es una placa infinita superconductora tipo II con anisotropía estructural o intrínseca, definida en el dominio $x \in[0, d],|y|>\infty,|z|>\infty$ en la geometría paralela, esto es, un campo magnético externo $\mathrm{H}_{\mathrm{e}}=H_{\mathrm{ey}} \hat{\mathrm{y}}+H_{\mathrm{ez}} \hat{z}$ incide sobre la placa en el plano $y-z$, de esta manera los campos electromagnéticos dependen solo de la variable $x$. Las ecuaciones de Maxwell asociadas a este sistema son:

$$
\begin{aligned}
& \mu_{0} j_{y}=-\partial_{x} B_{z} ; \mu_{0} j_{z}=\partial_{x} B_{y}, \\
& \partial_{t} B_{y}=\partial_{x} E_{z} ; \partial_{t} B_{z}=-\partial_{x} E_{y},
\end{aligned}
$$

se ha considerado que la variación del desplazamiento eléctrico es despreciable debido a que el campo magnético externo es de baja frecuencia y la condición de frontera $\mu_{0} \mathbf{H}=\mathbf{B}$ es válida porque se desprecia el primer campo crítico $\mathrm{H}_{\mathrm{c} 1}$. El corazón del modelo elíptico y sus variantes es la ecuación material $E=E(j)$, para nuestros propósitos tiene la forma explícita $\mathbf{E}=E \mathfrak{I} \bullet \mathbf{j}$, donde $\mathfrak{I}_{\mathrm{ij}}=\mathfrak{I}_{\mathrm{ci}} \boldsymbol{\delta}_{\mathrm{ij}}$, con $i, j=y, z$. De esta manera, las ecuaciones (I) quedan en términos sólo de la inducción magnética, en vista de que las componentes del tensor $\mathfrak{I}$ son:

$$
\begin{aligned}
& \mathfrak{J}_{c y}\left(B_{c}\right)=\frac{j_{0 y}}{\left(1+\frac{B_{c}}{B_{y}^{*}}\right)^{n_{y}}}, \\
& \mathfrak{J}_{c z}\left(B_{c}\right)=\frac{j_{0 z}}{\left(1+\frac{B_{c}}{B_{z}^{*}}\right)^{n_{z}}},
\end{aligned}
$$

$\mathrm{j}_{\mathrm{oy}}, \mathrm{j}_{\mathrm{oz}}, \mathrm{n}_{\mathrm{y}}, \mathrm{n}_{\mathrm{z}}, \mathrm{B}_{\mathrm{y}}^{*}, \mathrm{~B}_{\mathrm{z}}^{*}$, son parámetros de ajuste $\mathrm{y}$ $B_{c}$ es la inducción magnética en la placa superconductora en el estado crítico. La magnitud del campo eléctrico esta modelado por la ley vertical: Aquí la densidad de corriente crítica se obtiene de

$$
E= \begin{cases}0 & j \leq j_{c} \\ \rho\left(j-j_{c}\right) & j>j_{c}\end{cases}
$$

la relación material que tiene la forma:

$$
\frac{1}{j_{c}^{2}}=\left(\frac{\cos \phi_{c}}{\mathfrak{J}_{c y}\left(B_{c}\right)}\right)^{2}+\left(\frac{\operatorname{sen} \phi_{c}}{\mathfrak{J}_{c z}\left(B_{c}\right)}\right)^{2}
$$

donde $\phi_{c}$ es el ángulo que sustenta la densidad de corriente crítica respecto al eje y. Las ecuaciones (1-4) definen el modelo elíptico de estado crítico para estados cuasi-estacionarios. El objetivo es hallar el estado crítico del superconductor por lo que se emplea un algoritmo numérico, que a continuación se plantea.

\section{Metodología numérica}

Las ecuaciones de Maxwell (1) conforman un sistema de dos ecuaciones en derivadas parciales, para resolverlo se utilizó el método de líneas (MOL, (Schiesser, 2009)). Esta metodología numérica consiste en discretizar el dominio espacial tomando un conjunto de $\mathrm{N}$ puntos $\mathrm{x}_{1}, \mathrm{x}_{2}, \cdots, \mathrm{x}_{\mathrm{k}}, \mathrm{x}_{\mathrm{k}+1}, \cdots \mathrm{x}_{\mathrm{N}}$ equidistantes con tamaño de paso $\Delta x=x_{k+1}-x_{k}, k=1, N$. Las derivadas espaciales se aproximan con una fórmula de diferenciación numérica $D_{x} F\left(x_{k}\right)$ que transformara el sistema de dos ecuaciones a uno de $2 \mathrm{~N}$ ecuaciones diferenciales ordinarias. Para este trabajo se utilizaron tres fórmulas de diferenciación numérica con error de truncamiento de orden $0\left(h^{2}\right)$. Las ecuaciones de Maxwell tras la discretización toman la forma:

$$
\begin{aligned}
& \partial_{t} B_{y}\left(x_{k}\right)=+D_{x} E_{z}\left(x_{k}\right) \\
& \partial_{t} B_{z}\left(x_{k}\right)=-D_{x} E_{y}\left(x_{k}\right)
\end{aligned}
$$

Para simplificar la notación se definen las funciones evaluadas en los puntos $x_{k}$ como sigue: $F\left(x_{k}\right)=F^{k}$. Explícitamente, el sistema (5) luce como se muestra a continuación:

$$
\begin{aligned}
& \partial_{t} B_{y}^{1}=+\frac{-E_{z}^{3}+4 E_{z}^{2}-3 E_{z}^{1}}{2 \Delta x} \\
& \partial_{t} B_{y}^{k+1}=+\frac{E_{z}^{k+2}-E_{z}^{k}}{2 \Delta x}, k=1, N-2
\end{aligned}
$$




$$
\begin{aligned}
& \partial_{t} B_{y}^{N}=+\frac{3 E_{z}^{N}-4 E_{z}^{N-1}+E_{z}^{N-2}}{2 \Delta x} \\
& \partial_{t} B_{z}^{1}=-\frac{-E_{y}^{3}+4 E_{y}^{2}-3 E_{y}^{1}}{2 \Delta x} \\
& \partial_{t} B_{z}^{k+1}=-\frac{E_{y}^{k+2}-E_{z}^{k}}{2 \Delta x}, k=1, N-2 \\
& \partial_{t} B_{z}^{N}=-\frac{3 E_{y}^{N}-4 E_{y}^{N-1}+E_{y}^{N-2}}{2 \Delta x}
\end{aligned}
$$

Este sistema de ecuaciones diferenciales ordinarias se resolvió usando las bibliotecas estándar de MATLAB para ese propósito (L. F. Shampine, 2003). En este caso se utilizaron las rutinas ode23s y ode45, ya que el sistema es de los denominados Stiff: se utilizó ode23s para dar una solución gruesa y después se refinó con ode45. La distribución inicial de la inducción magnética al tiempo $\mathrm{t}=\mathrm{t}_{0}$ es $\mathbf{B}\left(\mathrm{x}, \mathrm{t}_{\mathrm{o}}\right)=0$. Para calcular las densidades de corriente $\mathrm{j}$ se utilizó un esquema similar al sistema (6) para preservar el error de truncamiento.

El paso de tiempo $\Delta$ t se aproximó con un análisis dimensional de la ecuación de Faraday. El criterio empleado es $\Delta t<t_{0}=B_{0} x_{0} / \rho j_{c o}$, donde $B_{o}, x_{0}, \rho j_{c o}$ son cantidades características de la inducción magnética, la distancia y el campo eléctrico, y se determinan de la situación física específica que se aborde.

El campo eléctrico se calcula usando la ley material y las ecuaciones (2-4):

$$
\begin{aligned}
& E_{y}^{k}=E^{k} \widetilde{J}_{c y}\left(B^{k}\right) j_{y}^{k}, \\
& E_{z}^{k}=E^{k} \widetilde{J}_{c z}\left(B^{k}\right) j_{z}^{k},
\end{aligned}
$$

donde se evalúan las ecuaciones (2) fuera del estado crítico en un campo $B^{k}$ a un tiempo $t$, y se utiliza la ley vertical como criterio de convergencia al rescribirse de la siguiente manera:

$$
E^{k}= \begin{cases}0 & j^{k} \leq j_{c}^{k} \\ \rho j_{c}^{k} \frac{\left(j^{k}-j_{c}^{k}\right)}{j_{c}^{k}} & j^{k}>j_{c}^{k}\end{cases}
$$

Esta ecuación es el punto medular del algoritmo, y plantea los siguientes hechos:

1. El campo eléctrico en estado estacionario se asume cero, sin embargo, el campo eléctrico está manejado por el error relativo $\left(j^{k}-j_{c^{k}}^{k}\right) / j_{c^{\prime}}^{k} y$ de acuerdo con ello, para que numéricamente $E^{k} \approx 0$, se deberá obtener $j^{k} \approx j_{c}^{k}$. La convergencia es lenta y por tanto los perfiles de $\mathbf{B}$ se hallan con un campo eléctrico pequeño pero finito.

2. La magnitud de la densidad de corriente generada por la ley de Ampere es:

$$
\left\|\mathbf{j}^{k}\right\|=\sqrt{\left[j_{y}^{k}\right]^{2}+\left[j_{z}^{k}\right]^{2}}
$$

y se compara durante todo el cálculo numérico con $j_{c}$ de la ecuación (4), utilizando la última, el ángulo $\phi^{\mathrm{k}}$ que sustenta $j^{\mathrm{k}}$ con el eje $\mathrm{y}$.

\section{Curvas de inducción magnética}

El modelo elíptico original (C. Romero-Salazar, 2003) ha sido exitoso al describir de manera cuantitativa resultados experimentales (I. F. VoIoshin, A. V. Kalinov, L. M. Fisher, S. A. Derevyanko, V. A. Yampol'skii, 2001). En el trabajo (C. Romero-Salazar, 2003) se estudió una placa superconductora de ytrio-bario-cobre-oxigeno (YBCO) y se obtuvieron los siguientes datos: los campos de penetración en dirección y y z, obtenidos de curvas de histéresis experimentales $\mu_{0} H_{\mathrm{Py}}=0.05 \mathrm{~T}$ y $\mathrm{H}_{\mathrm{Pz}}=0.25 \mathrm{~T}$; los valores óptimos de los parámetros de la ecuaciones (2) $n_{y}=0.5, n_{z}=0.22$, $\mathrm{B}_{\mathrm{y}}^{*}=0.02 \mathrm{~T}, \mathrm{~B}_{\mathrm{y}}^{*}=0.005 \mathrm{~T}$; la placa superconductora tiene un grosor de $\mathrm{d}=0.23 \times 10^{-3} \mathrm{~m}$; con estos valores se obtuvieron las densidades de corriente críti$\mathrm{ca} j_{\mathrm{oy}}=4.4842 \times 10^{9} \mathrm{~A} / \mathrm{m}$ y $j_{\mathrm{oz}}=5.0033 \times 10^{8} \mathrm{~A} / \mathrm{m}$.

La Figura 1 muestra perfiles de las componentes de la inducción magnética para un campo externo $\mathrm{H}_{\mathrm{e}}$ que incide sobre la placa superconductora con un ángulo de $65^{\circ}$ ocon respecto al eje $\mathbf{z}$. La magnitud del campo varía en $\mathrm{H}_{\mathrm{e}}=0.025,0.05$, $0.1,0.2,0.3,0.4,0.5,0.6$.

Los estados parcialmente penetrados $\left(H_{e}\right.$ no cubre la totalidad de la placa supercondúctora) corresponden a las magnitudes $\mathrm{H}_{\mathrm{e}}=\mathbf{0 . 0 2 5}$, $0.05,0.1$. Debido a la anisotropía estructural del material superconductor, la penetración del campo externo no se suscita de manera simultánea tal como se puede constatar en la mencionada figura. Las rutinas numéricas para resolver el problema de valor inicial requieren del intervalo de tiempo en el que se desea obtener la solución. El tiempo inicial se tomó como cero, mientras que el tiempo final es el tiempo requerido para establecer el estado estacionario. 


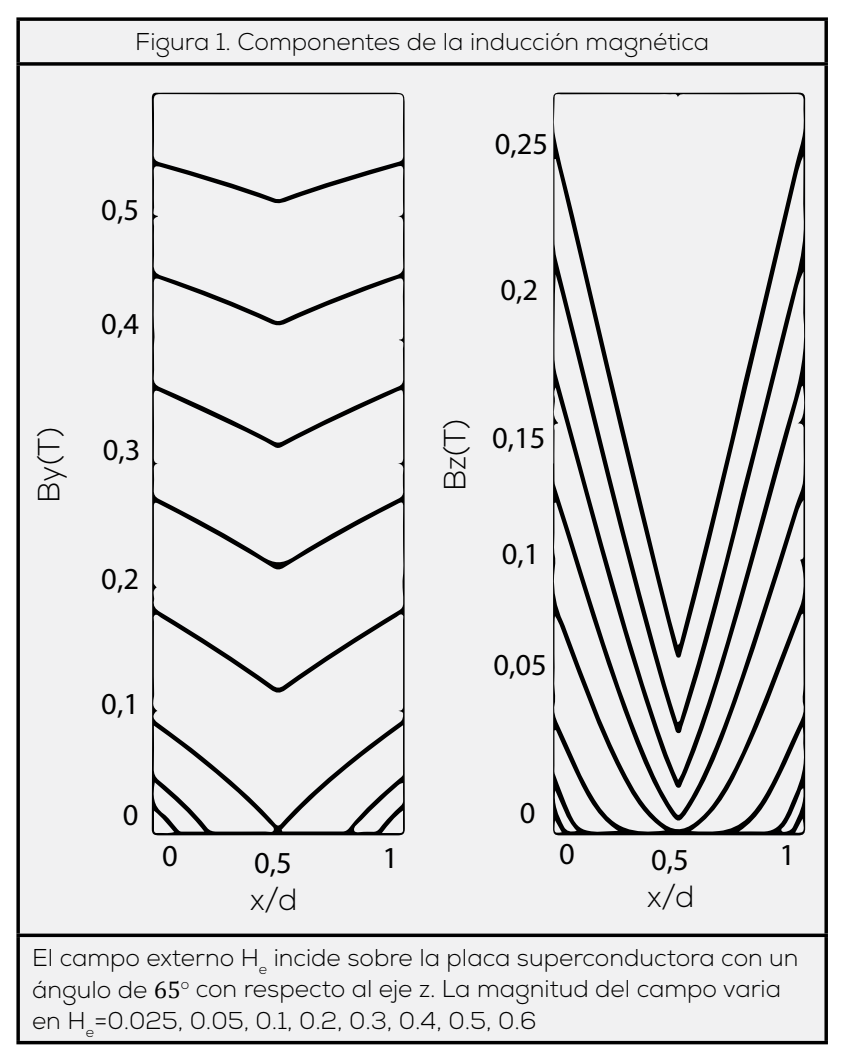

Para determinar numéricamente el estado estacionario se empleó como criterio de corte el error relativo medio $\mathrm{N}^{-1} \sum_{\mathrm{k}=1}^{\mathrm{N}}\left(\mathrm{j}^{\mathrm{k}}-\mathrm{j}_{\mathrm{c}}^{\mathrm{k}}\right) / \mathrm{j}_{\mathrm{c}}^{\mathrm{k}}$ a un tiempo determinado (tiempo final). Las curvas de la figura 1 corresponden a un tiempo de 12t_0, (los tiempos empleados por la rutina son menores), donde se consideró $B_{0}=1 T, x_{0}=d, j_{0}=j_{0 y}$. La Figura 2 muestra los cambios del error relativo al incrementar el tiempo, como se puede apreciar, a partir de 8 veces el tiempo característico el error relativo se mantiene sin variación significativa para estados penetrados. De manera evidente, dado que el error relativo de las curvas correspondientes a estados parcialmente penetrados es alrededor de $5 \times 10^{-1}$, se tiene que $j \approx 15 / 10 j$ lo que hace que las curvas de inducción magnética en mencionados estados, no sean fiables. En la figura 3 se muestra el campo eléctrico en el interior de la placa superconductora conforme se incrementa la magnitud del campo externo $\mu_{-} \mathrm{O} H$, ignorando los estados parcialmente penetrados, se estima para el campo eléctrico, para $\rho=10^{-8} \mathrm{Vm} / \mathrm{A}$, un valor aproximado de:

$$
E \approx \rho j_{0 y} \times \text { Error relativo } \approx 10^{-6} \mathrm{~V} / \mathrm{m}
$$
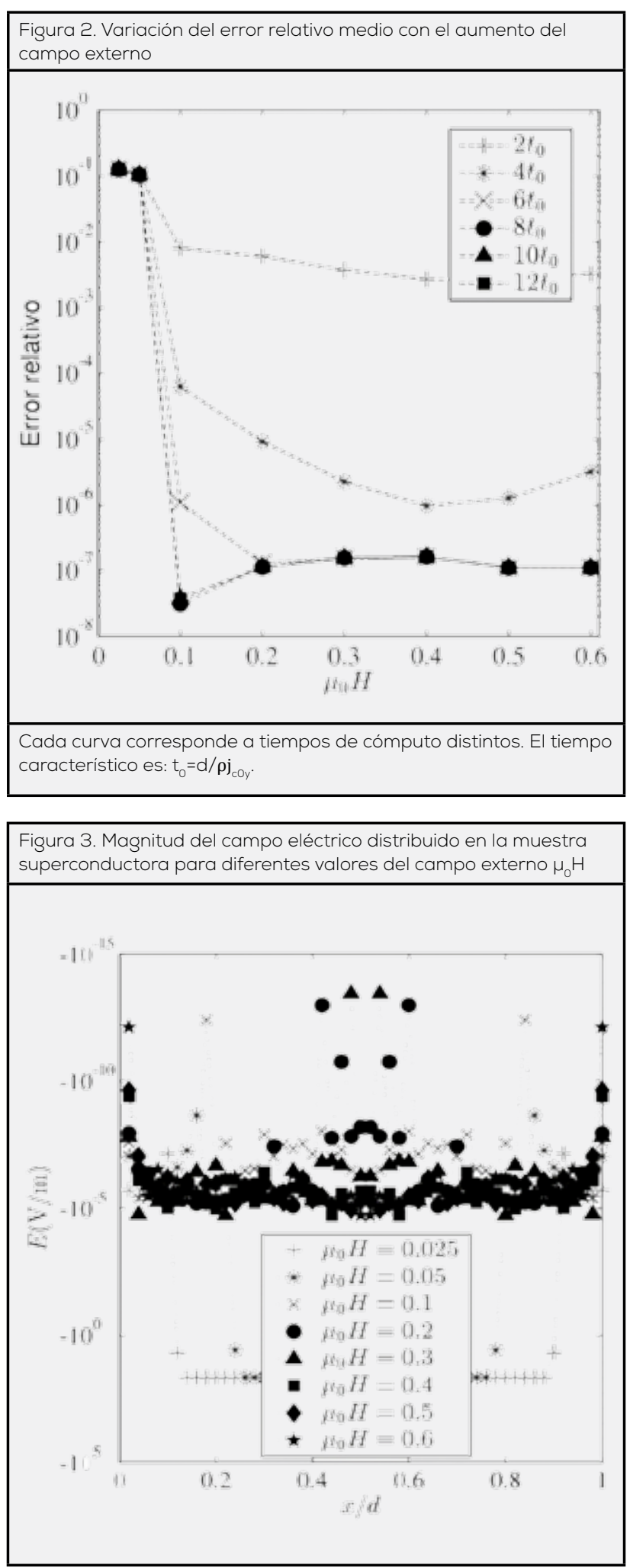
Esta estimación concuerda con los resultados numéricos de la curvas del campo eléctrico. Evidentemente el campo eléctrico dista de ser nulo o suficientemente pequeño para despreciar su presencia. Más aún, como se puede notar en la estimación realizada, la magnitud del campo eléctrico depende linealmente del valor de $\rho$

\section{Conclusiones}

La metodología numérica empleada, en general, arroja resultados fiables en los estados totalmente penetrados. Sin embargo, en los estados parcialmente penetrados la magnitud del campo eléctrico dista de ser nula, de modo especial, en la vecindad de la zona de Meissner. Un tratamiento especial en esas zonas requiere de análisis y estudio, en especial cuando se quiere estudiar frentes de flujo magnético e inestabilidades termomagnéticas.

Hasta donde llega nuestro conocimiento, otros autores que emplean la misma metodología numérica para resolver las ecuaciones de Maxwell para un superconductor tipo II en estado crítico, no realizan un análisis numérico de sus resultados, por lo que consideramos que este trabajo promueve que se tome particular atención en la vecindad de la zona de Meissner, cuando se implementen los algoritmos numéricos.

\section{Bibliografía}

Romero-Salazar, C., Pérez.-Rodríguez, Felipe. (2003). Critical state of anisotropic hard superconductors. Superconductor Science and Technology. Bristol, Reino Unido, p. 1273.

Schiesser, W. E. (2009). A Compendium of Partial Differential equations Models Method of Lines Analysis with Matlab. Cambridge University Press, Cambridge.

Shampine, L. F. y Main, I. G. (2003). Solving ODEs with MATLAB. Cambridge University Press, Cambridge. Voloshin I. F., Kalinov A. V., Fisher L. M. , Derevyanko, S. A., Yampol'skii, V. A. (2001). "A new type of peak effect in the magnetization of anisotropic superconductors". Journal of Experimental and Theoretical Physics Letters. Moscú pp. 285-288. 